\title{
Nonlinear Ultrasound Simulation Based on Full-Wave Model and Comparisons with KZK
}

\author{
Jinxin Zhao, Xingsong Li, Jinhua Yu, and Yuanyuan Wang
}

\begin{abstract}
The linear acoustic wave equation has been widely used for modeling the ultrasound propagation through biological tissues. However, the nonlinear effect can't be neglected when transmitting an ultrasound beam of high frequency or high amplitude. The commonly used nonlinear models for ultrasound imaging simulation include the KZK and the Full-wave model. This article firstly solves the full-wave equation numerically by using the Finite Difference Time Domain (FDTD) method. Experiments are then conducted to verify the accuracy of the numerical solution. A plane-wave imaging algorithm is consequently designed based on the full-wave simulation. The plane-wave imaging simulation based on the full-wave model can get the similar imaging quality for the simulation based on the KZK model with focused beam scanning mode. Simulation results prove that compared with KZK (CREANUIS) which is limited by the paraxial approximation, more flexible excitation setup and more feedback information can be acquired in the simulation based on the full-wave model and the FDTD. Thus it's helpful to better study nonlinear ultrasound.
\end{abstract}

Index Terms-Nonlinear propagation, full-wave, simulation, CREANUIS.

\section{INTRODUCTION}

The ultrasound propagation is a linear process under the assumptions of low frequency and small amplitude. In the past ten years, nonlinear ultrasound imaging technique has achieved a considerable development [1], [2]. It also leads to the presence of simulation methods for nonlinear ultrasound propagation [3]-[5]. The most commonly used model is the Khokhlov-Zabolotskaya-Kuznetsov (KZK) equation, which is a nonlinear one-way parabolic equation including the effect of attenuation, nonlinearity and attenuation [6]. However, the validity of the KZK is limited by the paraxial approximation to about $15 \sim 20^{\circ}$ from the axis of the ultrasound beam [7]. For this reason, other models such as the Westervelt equation [8] and the full-wave equation [7] have been proposed.

Traditionally the clinical ultrasound imaging devices use a focused beam to scan the tissue. In nonlinear ultrasound imaging, the excitation and imaging scheme may be more various. For example, for nonlinear parameter $B / A$ imaging, one main method is the pumping wave method [9], [10], which mixed a low-frequency pumping wave and a high-frequency probing beam [9]. Even a simple

Manuscript received February 25, 2014; revised April 28, 2014. This work is supported by The National Natural Science Foundation of China (61271071, 81101049), Shanghai Pujiang Talent Program (12PJ1401200) and Doctoral Fund of Ministry of Education (20110071 120019).

The authors are with the Department of Electronic Engineering of Fudan University, Shanghai, China, 200030 (e-mail: yywang@ fudan.edu.cn). single-frequency transmission method to measure the nonlinear parameter $B / A$ in homogeneous media may require a plane-wave emission [10]. Plane-wave imaging has also become an intensively researched topic in recent years because it can achieve the high imaging frame rate [11]. Since the KZK model has the paraxial limitation, it may not be an appropriate method to simulate plane-wave imaging.

This paper uses the full-wave model to simulate the plane-wave ultrasound imaging. The full-wave equation is numerically solved by using the finite difference time domain (FDTD) method. Experiments are then carried out to validate the accuracy of the numerical solution. The plane-wave imaging result generated from the full-wave model is finally compared with the one from the KZK model based on the focused beam scanning mode (CREANUIS [9]). Experiment results show that based on the full-wave model, more flexible excitation setup and more feedback information can be acquired in the simulation. This is more convenient for the study of nonlinear ultrasound.

The rest of this paper is organized as follows: the mathematical model and the numerical solution approach are first described. The imaging method based on the full-wave model and plane-wave is then introduced. Simulation results are consequently provided and compared with the results from the KZK. A discussion of the simulation and an outlook about the future work close the paper.

\section{METHODS}

\section{A. Full-Wave Model}

The full-wave equation describes the propagation of ultrasound through biological tissues with the effect of attenuation, nonlinearity, scattering, reflection and refraction [4], [7].

$$
\begin{aligned}
& \nabla^{2} p-\frac{1}{c_{0}^{2}} \frac{\partial^{2} p}{\partial t^{2}}+\frac{\delta}{c_{0}^{4}} \frac{\partial^{3} p}{\partial t^{3}}+\frac{\beta}{\rho c_{0}^{4}} \frac{\partial^{2} p^{2}}{\partial t^{2}}, \\
& +\frac{1}{\rho} \nabla p \cdot \nabla \rho-\sum_{m=1}^{v} \zeta_{m}=0
\end{aligned},
$$

while $\xi_{m}$ satisfies the following equation:

$$
\dot{\zeta}_{m}+\omega_{m} \zeta_{m}=a_{m} \omega_{m} \frac{\Delta c}{c_{0}} \nabla^{2} p
$$

In equation (1), $p$ is the acoustic pressure, $c_{0}$ and $\rho_{0}$ are the average sound velocity and the density of soft tissues, $\delta$ is the acoustic diffusivity, and $\beta$ is related to the nonlinear parameter $B / A$ with the equation $\beta=1+B / 2 A$. The last 
part in equation (1) stands for the relaxation mechanism which has $v$ peaks with each characteristic frequency $\omega_{m}$. The detail about selection of $\omega_{m}$ can be found in the paper of Pinton et al. [4].

The FDTD method is used to solve the full-wave equation [4]. The idea of the method is to discretize the space into lots of small grids and to calculate the pressure field of the whole simulated space. The pressure field is iteratively updated until the termination condition is reached. Since the simulation must be truncated into a finite space, some boundary conditions are necessary. Here we use the Perfectly Matched Layers (PML) to truncate the simulation field [4], [12]. PML can absorb the wave arriving at the boundary to avoid unwanted reflection which will bring error into the simulation. Both the echo data and the pressure field of any time step can be obtained. Thus it is possible for us to get the final B-mode image or to study the variation of the wave through its propagation. Compared with the simulation based on the KZK equation, the full-wave model with the FDTD numerical solutions allow more flexible excitation setup and information acquisition. A mixed programming of MATLAB and C language is introduced to get faster simulation.

\section{B. Imaging Simulation}

The main steps to get the simulated ultrasound images include: 1) setting the tissue properties and the scatterer distribution; 2) running the simulation program to get the RF echo data; 3) using filtering methods and beamforming strategy together with other post-processing steps (envelop detection and logarithm transformation) to get the final image. Since the full-wave model is not limited by the paraxial approximation as in the KZK model, more flexible excitation setup can be used. We design an imaging method based on the full-wave model by using plane wave. There are many post-beamforming strategies to enhance the quality of plane-wave imaging results. Here we just use the Hamming window as the apodization method after the calculation of the time delay for each imaging pixel. If the plane-wave imaging based on full-wave model can get the imaging quality similar to the one from the KZK model (with the same simulation parameter and post-processing method), it will be an indirect way to prove that full-wave model is a good tool to simulate plane-wave imaging in nonlinear ultrasound.

\section{EXPERIMENTS AND RESULTS}

All the simulations in this paper use a Gaussian-enveloped pulse with a $5 \mathrm{MHz}$ center frequency. In order to maintain stability in the computing process, the discretization grid side length is set as $0.025 \mathrm{~mm}$ and the sampling frequency is $123 \mathrm{MHz}$. Phantom definition should set the parameters $c, \rho, \delta$ and $\beta$ for every grid [7]. In homogeneous media, these parameters are set like this: $c=1540 \mathrm{~m} / \mathrm{s}, \rho=1000 \mathrm{Kg} / \mathrm{m}^{3}$, $\delta=0.5 \mathrm{~dB} / \mathrm{cm}$ and $\beta=5.5$ [7]. In other cases, some statistic models may be used to get the parameters of the simulated region, which usually causes discontinuity in the impedance. Some parameters of different tissues can be found in [7].

\section{A. Model Validation}

Three experiments are carried out to validate the simulation method used in this article. A simple experiment has first been done to see the difference of the fundamental and harmonic ultrasound beams. The length of the array is $2.5 \mathrm{~cm}$ and the focal length is $3 \mathrm{~cm}$. The excited sound pressure of each element is set to be $1 \mathrm{Mpa}$. Simulated tissues are homogeneous. The wave data of each point in the pressure field is recorded and then the amplitude distributions of different frequency components are drawn.

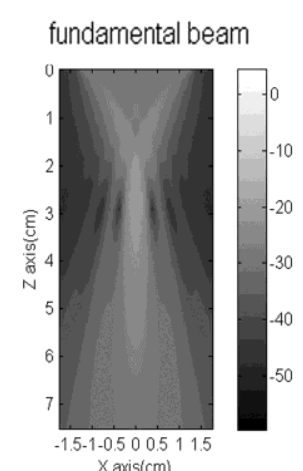

(a)

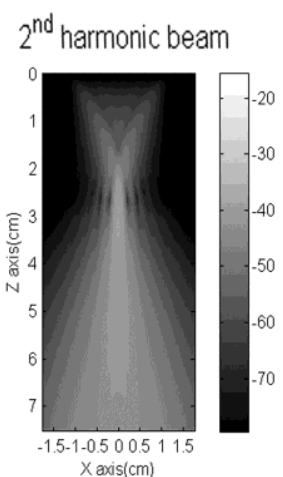

(b)

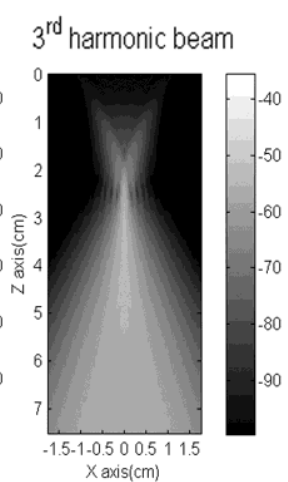

(c)
Fig. 1. Beam pattern of different components: (a) the fundamental (b) the second harmonic (c) the third harmonic. The images are proposed in $\mathrm{dB}$ scale using the maximum pressure of the fundamental beam.

Fig. 1 shows the beam pattern of different frequency components. It is obvious that the fundamental beam is wider than the 2nd harmonic ultrasound beam, while the 3rd harmonic is narrower than the 2 nd harmonic. The main lobe widths are respectively $7.75 \mathrm{~mm}, 4.25 \mathrm{~mm}$ and $3.00 \mathrm{~mm}$ for the fundamental, second and third harmonic beams .This corresponds with the theory that higher frequency beam has narrower width for its smaller wavelengths [2]. The difference of color bar in Fig. 1 tells the amplitude difference of different frequency components. The maximum pressures are $2.31 \mathrm{Mpa}$, $0.27 \mathrm{Mpa}$ and $0.048 \mathrm{Mpa}$, respectively, in accordance with the view that energies transmit from lower frequency harmonics to higher frequency harmonics. The fundamental maximum pressure is higher than the excitation pressure of each element for the focal effect. Fig. 1 also shows that since the harmonic components are generated through the beam propagation, the near field energy of the harmonic components is quite small. That's an advantage of second harmonic imaging for it can avoid near field aberration and enhance the image quality [7].

$B / A$ is an important parameter in nonlinear acoustics. It shows great potential applications in nonlinear ultrasound imaging. Here we use our simulation for a $B / A$ determination in homogeneous medium. The method is based on single-frequency transmission with direct mode [10]. The amplitude of the transmitted pulse is $0.1 \mathrm{Mpa}$. More details about the mathematical methods could be found in [10], [13]. Since the full-wave simulation can record the waveform of any point in the simulation area and thus we can get the waveform through the wave propagation way, then to calculate $B / A$. The $B / A$ parameter is set to be 5.2, 9.0 and 12.0 in three simulated situations. The determination results based on the directed mode model [10], [13] are respectively 5.33, 9.20 and 12.25. All the results are within the error range of $3 \%$ compared to the true value, which indicates the accuracy of the nonlinear wave effect simulation in this article. 


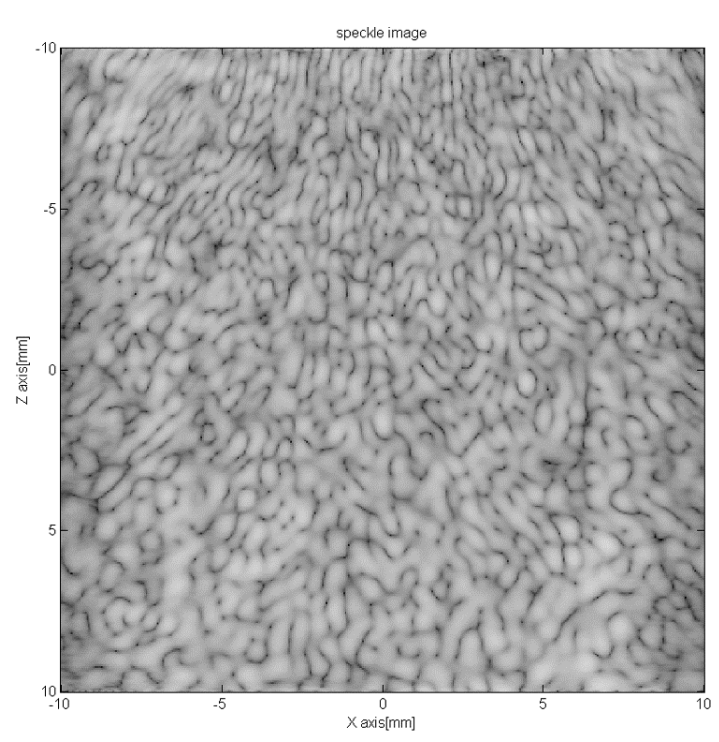

(a)

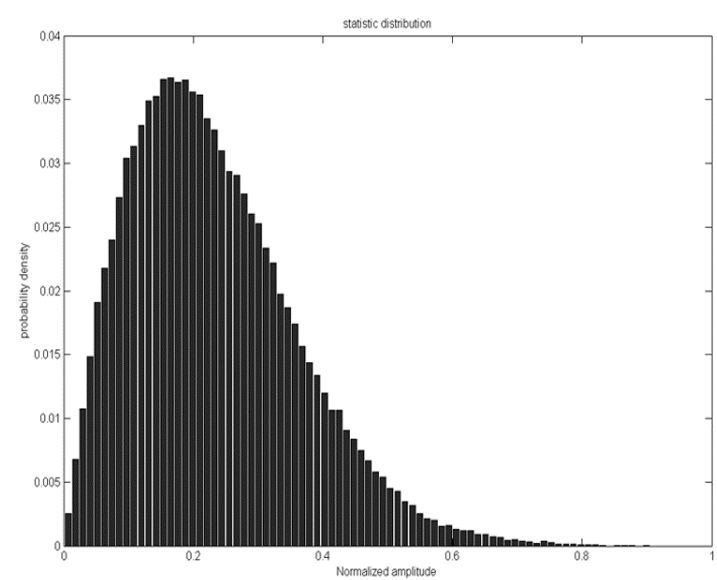

(b)

Fig. 2. (a) the speckle patterns of a large number of scatterers (b) the statistic distribution which corresponds to the Rayleigh distribution. The variance $\sigma$ in Rayleigh distribution expression of the fitting result is 0.0056 .

To verify the accuracy of the statistic characteristic of the ultrasound image, a homogeneous medium containing scatterers with random amplitude (maximum 5\% difference in acoustic impedance from the surrounding medium) and random spatial position is modeled. No other target is in the medium. Plane-wave mode is used to get the speckle pattern. The normalized amplitude of the speckle is expected to fit a
Rayleigh distribution:

$$
f(x)=\frac{x}{\sigma^{2}} \exp \left(-\frac{x^{2}}{2 \sigma^{2}}\right)
$$

Fig. 2 shows the speckle pattern and the statistic amplitude distribution result. The envelope is expected to follow the Rayleigh distribution for it is the norm of a random two-dimensional vector with its two components independent and having the same variance. From Fig. $2 b$, the result is accordant to the literature $(\sigma=0.0056)$.

\section{B. Plane-Wave Imaging}

We simulate a cyst phantom imaging by using plane-wave and compare the result with the image simulated by CREANUIS, which is based on the KZK equation [14]. CREANUIS is a nonlinear ultrasound simulator, which generates US images by repeating the same basic operations for each simulated line [14]. It first uses GASM to compute the nonlinear US field and then calculate the backscattered echo by accumulation of the effect of each scatterer [14]. Thus the computational amount is quite proportional to the number of scatterers.

The cyst phantoms are two series of five circular areas, one with high intensity scatterers and another with no scatterers. The number of scatterers is set to be 3 per $\mathrm{mm}^{3}$. In the CREANUIS simulation we use a focused beam and 300 scanning lines to get the echo data and finally to form the US image. The Hamming window is used in both transmitting and receiving for apodization. In simulation based on the full-wave, plane wave imaging is used. All the source elements transmit the pulse at the same time to form a plane wave. The Hamming window is also used as the apodization strategy in the receiving to obtain the final image. Simulation time step and discretization size are the same as said before.

Fig. 3 shows the images generated by CREANUIS and by our simulation based on full-wave equation. Because the plane-wave imaging doesn't focus the energy in pulse emission, the lateral resolution and contrast will be worse to some extent even though the other parameters are the same. Here we see the results of the CREANUIS and of our simulation are similar, which validates of the full-wave model to simulate plane-wave imaging.

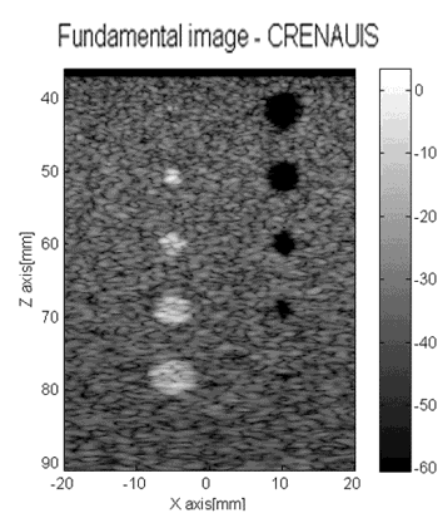

(a)

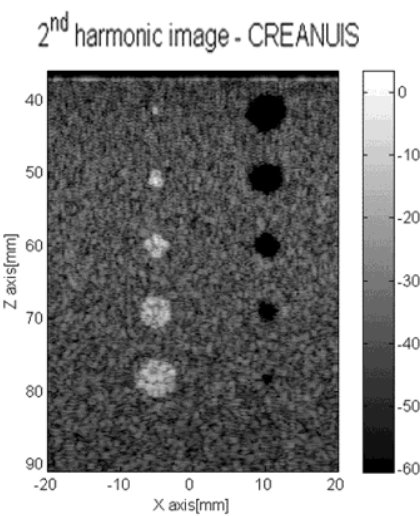

(b)

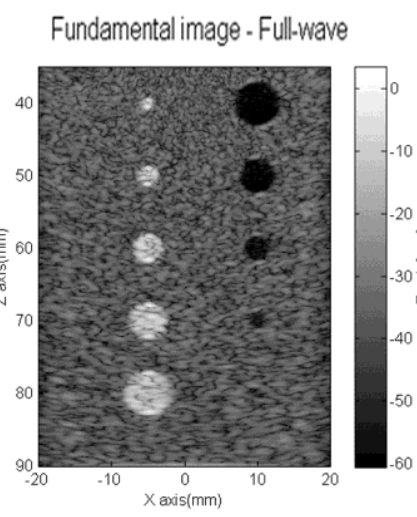

(c)

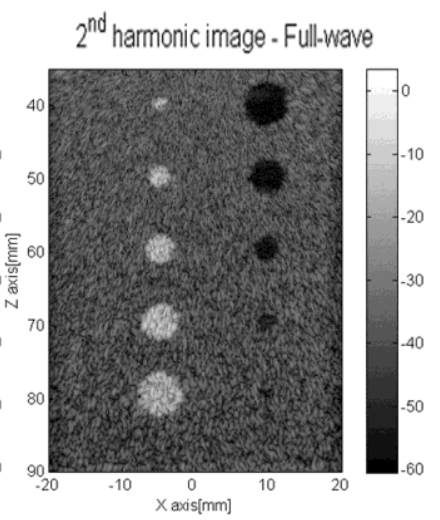

(d)

Fig. 3. Image simulation with the CREANUIS (a) and (b) and with the full-wave model (c) and (d), (a) and (c) are the fundamental images, (b) and (d) are the second harmonic images. 


\section{CONCLUSION}

We use the full-wave equation to simulate a plane-wave nonlinear ultrasound imaging process in this paper. In recent years, research work on nonlinear ultrasound is thriving. It shows great potential in clinical use. Meanwhile, plane-wave imaging also restarted to catch researchers' interests for the development in graphical processing unit (GPU)-based platforms have made clinical ultrafast ultrasound imaging possible. The work of this paper provides an efficient tool for studying in this field. This paper firstly illustrates the nonlinear ultrasound propagation simulation based on the full-wave model and then uses the simulation method in the study of harmonic imaging and nonlinear parameter determination to validate our simulation accuracy. The advantage of the full-wave model is that during the simulation, the accurate wave pressure data of any point in the simulated region is calculated and can be obtained. This makes it possible to simulate nonlinear plane-wave imaging, while the KZK equation is constrained by paraxial approximation. Results show that the image generated by this model using the plane-wave scanning mode is similar to those generated by simulation based on KZK equation. As the full-wave model allows more flexible excitation setup and data acquisition, it can help to deeply study the nonlinear wave effects in different medium and to find the key of nonlinear imaging.

Next we plan to apply some program acceleration techniques to run the simulation faster. $\mathrm{C}++$ or GPU technologies may help. Other ideas include using angular spectrum approach or other methods to solve the full-wave equation [15], because the FDTD method is strictly restricted by the contradiction of the accuracy and computation amount. Future work will be focused on the techniques of nonlinear ultrasound imaging, such as pulse coding and beamforming strategy, based on this paper's simulation.

\section{REFERENCES}

[1] R. S. Shapiro, A. Stancato-Pasik, and S. E. Sims, "Diagnostic value of tissue harmonic imaging compared with conventional sonography," Computers in Biology and Medicine, vol. 35, issue 8, pp. 725-733, October 2005.

[2] F. A. Duck, "Nonlinear acoustic in diagnostic ultrasound," Ultrasound in Med. \& Biol., vol. 28, no.1, pp.1-18, January 2002.

[3] Y. Li and J. A. Zagzebski, "Computer model for harmonic ultrasound Imaging," IEEE Trans. Ultrasonics, Ferroelectrics, and Frequency Control, vol. 47, no. 4, pp.1000-1013, July 2000.

[4] G. F. Pinton, J. Dahl, S. Rosenzweig, and G. E. Trahey, "A heterogeneous nonlinear attenuating full-wave model of ultrasound," IEEE Trans. Ultrasonics, Ferroelectrics, and Frequency Control, vol. 56, no. 3, pp.474-488, March 2009.

[5] T. Huttunen, M. Malinen, and J. P. Kaipio, "A full-wave helmholtz model for continuous-wave ultrasound transmission," IEEE Trans. Ultrasonics, Ferroelectrics, and Frequency Control, vol. 52, no. 3, pp. 397-409, March 2005.

[6] V. F. Humphrey, "Nonlinear propagation in ultrasonic fields: measurements, modeling and harmonic imaging," Ultrasonics, vol. 38, Issue 1-8, pp. 267-272, March 2000.

[7] G. F. Pinton, G. E. Trahey, and J. J. Dahl, "Sources of image degradation in fundamental and harmonic ultrasound imaging using nonlinear, full-wave simulations," IEEE Trans. Ultrasonics, Ferroelectrics, and Frequency Control, vol. 58, no. 4, pp.754-765, April 2011.
[8] A. Karamalis, W. Wein, and N. Navab "Fast ultrasound image simulation using the westervelt equatioin," Lecture Notes in Computer Science 2010, vol. 6361, pp. 243-250.

[9] N. Ichida, T. Sato, H. Miwa, and K. Murakami "Real-time nonlinear parameter tomography using impulsive pumping waves," IEEE Trans. Sonics and Ultrasonics, vol. Sub.31, no. 6, pp. 635-641, November 1984.

[10] F. Varray, O. Basset, P. Tortoli, and C. Cashard, "Extensions of nonlinear B/A Parameter imaging methods for echo mode," IEEE Trans. Ultrasonics, Ferroelectrics, and Frequency Control, vol. 58, no. 6, pp. 1232-1244, June 2011.

[11] G. Montaldo, M. Tanter, J. Bercoff, N. Benech, and M. Fink, "Coherent plane-wave compounding for very high frame rate ultrasonography and transient elastography," IEEE Trans. Ultrasonics, Ferroelectrics, and Frequency Control, vol. 56, no. 3, March 2009.

[12] X. Li, "PML condition for the numerical simulation of acoustic wave," in Proc. 2010 International Conf. Computing, Control and Industrial Engineering, 2010, vol. 2, pp.129-132.

[13] W. K. Law, L. A. Frizzell, and F. Dunn, "Determination of the nonlinear parameter B/A of biological media," Ultrasound in Med. \& Biol., vol. 11, no.2, pp. 307-318, March-April 1985.

[14] F. Varray, P. Tortoli, O. Basset, and C. Cashard, "CREANUIS: A nonlinear radiofrequency ultrasound image simulator," Ultrasound in Med. \& Biol., vol. 39, no. 10, pp. 1915-1924, 2013.

[15] Y. Du and J. A. Jensen, "Fast simulation of non-linear pulsed ultrasound fields using an angular spectrum approach," Ultrasonics vol. 53, issue 2, pp. 588-594, February 2013.

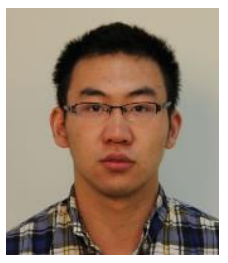

Jinxin Zhao was born in Yangzhou of Jiangsu Province, China in 1990. He received a B.S. degree of electronic engineering from Fudan University, Shanghai, China, in 2012 and is now a PHD candidate in the Department of Electronic Engineering in Fudan University. His research mainly focuses on ultrasound imaging.

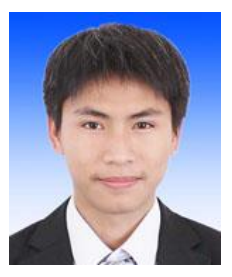

Xingsong Li was born in Xingyi City, Guizhou Province, China in 1989. He received the B.S. degree from Fudan University, Shanghai, China, in 2012. He is currently pursuing the M.S. degree in electronic engineering in Fudan University, Shanghai, China. His research interests are ultrasound imaging.

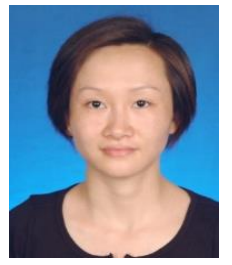

Jinhua Yu received the Ph.D. degree from Fudan University, Shanghai, China, in 2008. She was a postdoctoral fellow at the University of Missouri, USA from 2008 to 2010. She is currently an associated researcher of Fudan University. Her current research interests include medical image analysis and ultrasound imaging.

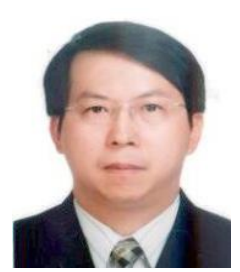

Yuanyuan Wang received the B.Sc., M.Sc. and Ph.D. degrees in electronic engineering from Fudan University, Shanghai, China, in 1990, 1992 and 1994, respectively.

During 1994 to 1996, he was a postdoctoral research fellow with the School of Electronic Engineering and Computer Science at University of Wales, Bangor, UK. In May 1996, he went back to Department of Electronic Engineering at Fudan University as an associate professor. He was then promoted to a full professor in May, 1998. He is currently the director of Biomedical Engineering Center and vice dean of Information Science and Engineering School at Fudan University. He is also the author or coauthor of 6 books and 400 research papers. His research interests include medical ultrasound techniques and medical signal processing. 